\title{
A Cdh1-FoxM1-Apc axis controls muscle development and regeneration
}

\author{
Zhe Chen', Lei Li', Shuangnian Xu', Zhilong Liu', Chengfang Zhou', Zhigang Li', Yuanyuan Liu', Weiru Wu', \\ Yongxiu Huang ${ }^{1}$, Mei Kuang ${ }^{1}$, Shijun Fan ${ }^{2}$, Hui Li ${ }^{1}, \mathrm{Xi} \mathrm{Li}^{1}$, Guanbin Song ${ }^{3}$, Wen-Shu Wu${ }^{4}$, Jieping Chen ${ }^{1}$ and Yu Hou ${ }^{1}$
}

\begin{abstract}
Forkhead box M1 (FoxM1) transcriptional factor has a principal role in regulating cell proliferation, self-renewal, and tumorigenesis. However, whether FoxM1 regulates endogenous muscle development and regeneration remains unclear. Here we found that loss of FoxM1 in muscle satellite cells (SCS) resulted in muscle atrophy and defective muscle regeneration. FoxM1 functioned as a direct transcription activator of adenomatous polyposis coli (Apc), preventing hyperactivation of wnt/ $\beta$-catenin signaling during muscle regeneration. FoxM1 overexpression in SCS promoted myogenesis but impaired muscle regeneration as a result of spontaneous activation and exhaustion of SCS by transcriptional regulation of Cyclin B1 (Ccnb1). The E3 ubiquitin ligase Cdh1 (also termed Fzr1) was required for FoxM1 ubiquitylation and subsequent degradation. Loss of Cdh1 promoted quiescent SCs to enter into the cell cycle and the SC pool was depleted by serial muscle injuries. Haploinsufficiency of FoxM1 ameliorated muscle regeneration of Cdh1 knock-out mice. These data demonstrate that the Cdh1-FoxM1-Apc axis functions as a key regulator of muscle development and regeneration.
\end{abstract}

\section{Introduction}

Skeletal muscle is a homeostatic tissue that requires myogenesis upon postnatal growth and muscle regeneration following an injury ${ }^{1}$. Myogenesis is a tightly controlled process including muscle stem cells (also called muscle satellite cells (SCs)), which proliferate, differentiate to myoblasts, and subsequently form multinucleated myotubes ${ }^{2,3}$. A portion of activated SCs return to quiescence and replenish the SC pool ${ }^{4}$. The balanced transition of SCs between quiescence, proliferation, and differentiation is critical for SC maintenance and muscle homeostasis.

\footnotetext{
Correspondence: Jieping Chen (chenjpxn@163.com) or

Yu Hou (houyuxn@vip.126.com)

'Department of Hematology, Southwest Hospital, Third Military Medical University (Army Medical University), 400038 Chongqing, China

${ }^{2}$ Medical Research Center, Southwest Hospital, Third Military Medical University (Army Medical University), 400038 Chongqing, China

Full list of author information is available at the end of the article.

These authors contributed equally: Zhe Chen, Lei Li

Edited by G. Blandino
}

Muscle-specific transcriptional factors, including Pax3, Pax7, Mfy5, MyoD, and Myogenin, play critical roles in the determination of the fate of SCs, as well as in muscle development and regeneration ${ }^{5}$. Several key components of SC niche, such as laminin- $\alpha 1$ and collagen VI, are necessary for self-renewal of SCs and muscle regeneration $^{6,7}$. Notch signaling, as well as p38/mitogen-activated protein kinase, wnt, phosphatase and tensin homolog, and several cell cycle regulators have been shown to regulate SC expansion and muscle development ${ }^{8-11}$. Recently, the ubiquitin-proteasome system has been demonstrated to be essential for the maintenance of SCs. Knockout of the Rp13 proteasomal component impairs the ability of SCs to proliferate, survive, and differentiate, resulting in defective muscle regeneration ${ }^{12}$. The anaphase-promoting complex/cyclosome (APC/C) is a multisubunit E3 ubiquitin ligase complex that regulates cyclic degradation of cell cycle regulator via the $\mathrm{Cdh} 1$ or $\mathrm{Cdc} 20$ adaptor proteins ${ }^{13}$. APC/C $\mathrm{C}^{\mathrm{Cdh} 1}$ has a broad spectrum of substrates in and beyond the cell cycle ${ }^{14,15}$. However, the role of APC/ $\mathrm{C}^{\mathrm{Cdh} 1}$ in SC maintenance is unclear. 
Forkhead box M1 (FoxM1) is one of the substrates of $\mathrm{APC} / \mathrm{C}^{\mathrm{Cdh} 1}$ in several cancer cells ${ }^{16,17}$ and is a wellestablished regulatory transcriptional factor for cell cycle progression ${ }^{18}$. FoxM1 has been reported to play critical roles in stem cells ${ }^{19,20}$. FoxM1 is essential for proliferation of human embryonic stem cells and protects them against oxidative stress by transcriptionally regulating Cyclin B1 (Ccnb1) and cyclin-dependent kinase $1(\mathrm{Cdk} 1)^{19}$. The higher expression of FoxM1 is essential for the maintenance of hematopoietic stem cells by transcriptionally regulating Nurr $1^{20}$. We previously described that FoxM1 promotes the proliferation and survival of $\mathrm{C} 2 \mathrm{C} 12$ cells (an immortalized myoblast cell line) in vitro partially by the involvement of long noncoding RNAs ${ }^{21}$. FoxM1 expression displays dynamic changes during the transition of $\mathrm{C} 2 \mathrm{C} 12$ between quiescence and activation ${ }^{21}$, implying the important roles of FoxM1 in SCs. However, the precise functions of FoxM1 in SCs and whether FoxM1 protein is a substrate of $\mathrm{APC} / \mathrm{C}^{\mathrm{Cdh} 1}$ are largely unknown.

Besides its transcriptional regulation of target genes, FoxM1 has been reported to directly interact with proteins, such as $\mathrm{Npm}^{22}, \mathrm{Rnf168}^{23}, \mathrm{Melk}^{24}$, and beta $(\beta)$ catenin $^{25,26}$. $\beta$-Catenin is the core factor of the wnt pathway. Degradation of $\beta$-catenin is promoted by a cytoplasmic destruction complex consisting of adenomatous polyposis coli (Apc), Axin, Cki, and Gsk3 $\beta$, which phosphorylates $\beta$-catenin ${ }^{27}$. Conversely, FoxM1 binds directly to $\beta$-catenin and enhances $\beta$-catenin nuclear localization in glioma cell lines ${ }^{25}$ and pancreatic cancer cells $^{26}$. The constitutive activation of $\beta$-catenin in mice has no obvious effect on the amount of SCs under homeostatic conditions, but these mice show muscle regeneration defects with aberrant myofiber morphology and atrophy, which is mainly attributed to hyperactive transforming growth factor-beta (TGF- $\beta$ ) signaling ${ }^{28}$. However, little is about the role of FoxM1 in muscle regeneration and the correlation between FoxM1 and wnt signaling in SCs.

In this study, we demonstrate that the loss of FoxM1 results in muscle atrophy and damaged muscle regeneration. Both FoxM1 deficiency and overexpression impaired SC maintenance. Unexpectedly, FoxM1 promoted $\beta$-catenin degradation by transcriptionally regulating Apc. Moreover, the accumulation of FoxM1 protein was controlled by $\mathrm{Cdh} 1$ via ubiquitylation. The identified Cdh1-FoxM1-Apc regulatory axis provides new insight on the regulators of SC maintenance and muscle homeostasis.

\section{Results \\ FoxM1 deficiency results in muscle atrophy and impairs muscle regeneration}

To determine whether FoxM1 regulates muscle homeostasis, we crossed mice with loxP-flanked FoxM1 alleles $\left(\text { FoxM } P^{f l f f}\right)^{29}$ with Pax7-Cre mice ${ }^{30}$ to generate control FoxM1 $1^{f l f l}$ mice and FoxM1 $1^{f l f l}$ Pax $7-C r e$ mice (designated here as "FoxM1-cKO mice") (Supplementary Fig. S1a, b). The expression of FoxM1 in SCs was efficiently knocked out (Supplementary Fig. 1c, d). The weight of tibialis anterior (TA) muscle in FoxM1-cKO mice showed no obvious differences with $F o x M 1^{f l / f l}$ mice at 2 months of age but showed decreased weight at 8 months of age (Fig. 1a, b and Supplementary Fig. 1e). Moreover, the myofibers of extensor digitorum longus (EDL) in $F o x M P^{f l / f l}-c K O$ mice showed reduced numbers of myonuclei/myofiber than FoxM1 $1^{f / f l}$ mice at 8 months of age (Fig. 1c, d and Supplementary Fig. 1f). Histological analysis of TA revealed muscle atrophy with age in FoxM1-cKO mice compared with control mice (Fig. 1e, f and Supplementary Fig. 1g). FoxM1-cKO mice were inferior in the maximum running distance compared with control mice at 8 months of age (Fig. 1g and Supplementary Fig. 1h). These data suggested that loss of FoxM1 in SCs resulted in muscle loss with age.

To explore the effect of FoxM1 deficiency on muscle regeneration, we induced muscle injury by injecting $\mathrm{BaCl}_{2}$ into muscles of mice at 2 months of age (Fig. 1h) Histological analysis of the TA muscles at 7 days and 14 days after injury revealed a more severe regeneration defect in FoxM1-cKO mice, as evidenced by the larger unrepaired areas (Fig. 1i). Deletion of FoxM1 in SCs resulted in smaller-sized regenerative myofibers, compared with control mice at 14 days post-injury (DPI) (Fig. 1j). Together, these data suggested that loss of FoxM1 in SCs resulted in muscle atrophy and impaired muscle regeneration.

\section{FoxM1 deficiency impairs SC maintenance by impeding cell cycling of SCs}

The finding of decreased muscle mass in FoxM1-cKO mice prompted us to examine the abundance of SCs in the skeletal muscle. Since SCs have definite cell surface markers (defined as $\mathrm{CD} 45^{-} \mathrm{Sca}^{-} \mathrm{CD} 11 \mathrm{~b}^{-} \mathrm{CD} 31^{-} \mathrm{CD} 34^{+} \alpha$ 7 -integrin $\left.{ }^{+}\right)^{31}$, we utilized flow cytometry to analyze the SC pool. FoxM1 deletion had no obvious effect on the abundance of SCs in 2-month-old mice (Supplementary Fig. 2a) but considerably reduced SCs abundance in 8-month-old mice (Fig. 2a, b). Immunostaining revealed considerably fewer numbers of $\mathrm{Pax}^{+}$SCs per fiber in 8-month-old FoxM1-cKO mice than in their control littermates (Fig. 2c, d). These data suggested that loss of FoxM1 impaired SC maintenance.

We analyzed the cell cycle of SCs and observed a significantly increased frequency of quiescent SCs in FoxM1-cKO mice (Fig. 2e, f). We also found a decreased percentage of S phase SCs in FoxM1-cKO mice compared with control mice (Fig. 2g, h). In vitro analysis showed that deletion of FoxM1 inhibited the incorporation of 


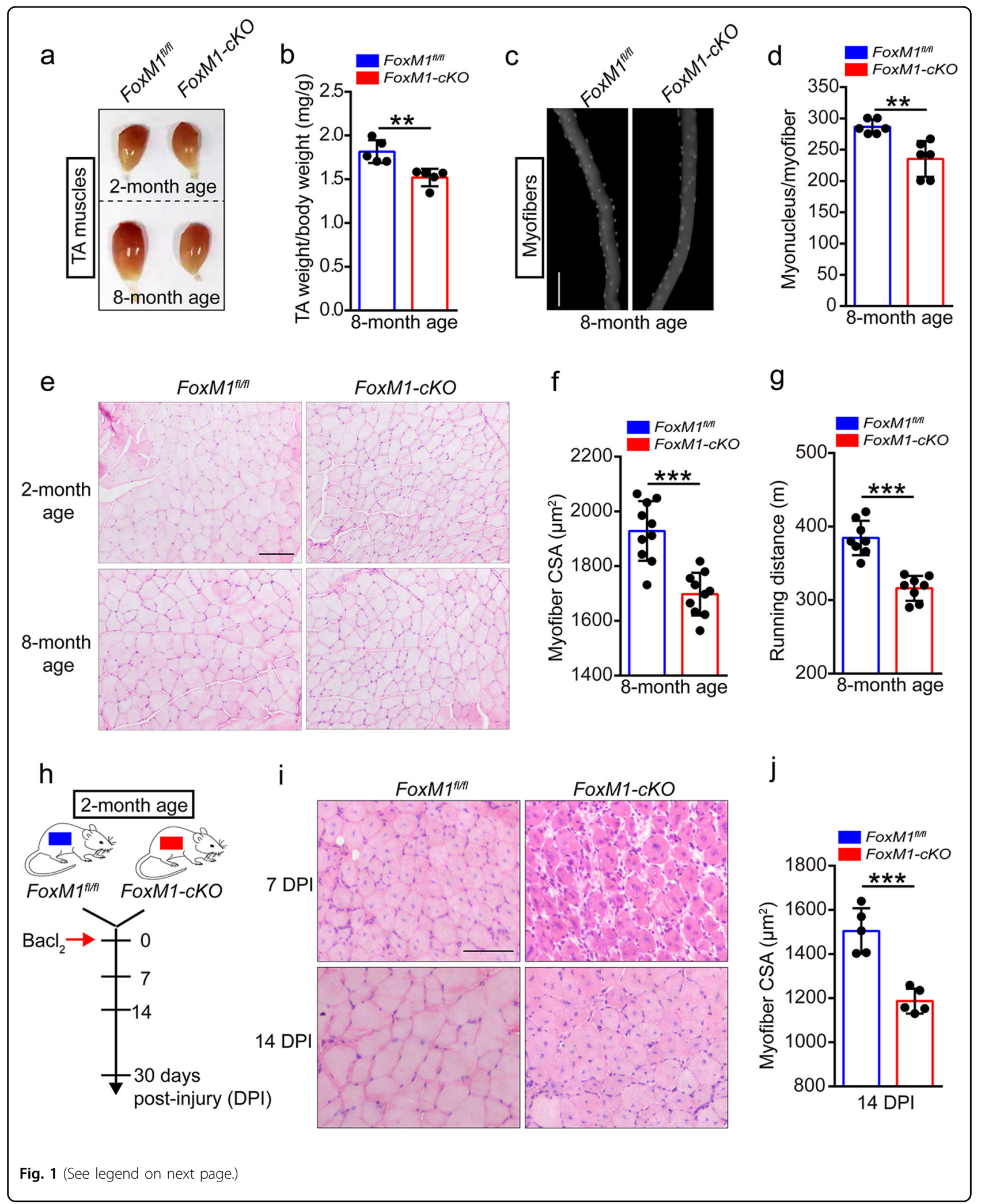


(see figure on previous page)

Fig. 1 FoxM1 deficiency results in muscle atrophy and impairs muscle regeneration. a The visual comparison of muscle mass of tibialis anterior (TA) in FoxM1 deletion mice compared with control mice at 2 or 8 months of age. b Quantification of TA weight/body weight in FoxM $7^{f / / f}$ and FoxM1-CKO mice at 8 months of age $(n=5)$. c, $\mathbf{d}$ Immunofluorescence of myonucleus on single myofiber by DAPI staining (c) and number count (d) ( $n=6,20$ myofibers per mouse). Scale bar, $100 \mu \mathrm{m}$. e Hematoxylin and eosin (HE) staining of the TA muscle cross-sections in mice at 2 or 8 months of age. Scale bar, $100 \mu \mathrm{m}$. f Average cross-section area (CSA) of TA in FoxM1 $7^{\text {fl/f }}$ and FoxM1-cKO mice at 8 months of age $(n=10)$. $\mathbf{g}$ The maximum running distance (measured by a treadmill) of FoxM1-cKO mice compared with control mice at 8 months of age $(n=8)$. $\mathbf{h}$ A schematic illustration showing the experimental design for muscle injury. $\mathrm{BaCl}_{2}$ was injected into and along the length of TA and gastrocnemius muscle of mice (2 months of age). The skeletal muscle or TA muscle was harvested at 7-30 days post-injury (DPI) depending on the experiments. $\mathbf{i}$ HE staining of the TA muscle cross-sections of FoxM1-CKO mice compared with control mice at 7 days and 14 DPI. Scale bar, $100 \mu \mathrm{m}$. j Average CSA of TA muscle in mice at 14 DPI $(n=5)$. Error bars represent the means \pm SD. ${ }^{* *} p<0.01,{ }^{* *} p<0.001$; Student's $t$ test.

bromodeoxyuridine (BrdU) and expansion of SCs (Supplementary Fig. 2b, c and Fig. 2i). Loss of FoxM1 may result in cell apoptosis. Deletion of FoxM1 resulted in a slight increase in apoptosis (Fig. 2j). The differentiation potential of SCs is vital for muscle regeneration. However, we found that the loss of FoxM1 had no obvious effect on SC differentiation (Supplementary Fig. 2d-f). Together, these data suggested that FoxM1 was essential for SC maintenance by regulating the proliferation and survival of SCs.

\section{Loss of FoxM1 results in activation of wnt/ $\beta$-catenin signaling in $\mathrm{SCs}$}

We performed global gene expression profiling of SCs isolated from 2-month-old FoxM1-cKO mice that were uninjured or $48 \mathrm{~h}$ post-injury. We found that 141 genes had over twofold differential expression in FoxM1-cKO SCs relative to their expression in FoxM $1^{f l f l} \mathrm{SCs}$ in uninjured mice, In injured mice, 515 genes were differentially expressed (Fig. 3a, b). Notably, cell cycle regulation and the ribosome pathway were the most commonly modified cellular function pathways in FoxM1-cKO SCs under homeostatic status, and p53, Foxo, and wnt pathways were enriched in injured mice (Fig. 3c, d). The differential expression of 28 genes, including the cell cycle regulator, Ccnb1, occurred in both the homeostatic and injured conditions (Fig. 3e). These results revealed that FoxM1 promoted cell cycling of SCs at the molecular level.

We also found genes of wnt signaling pathway that displayed downregulated expression in SCs in injured mice. These genes included Apc, Gsk3 $\beta$, Axin1, and Skpla (Fig. 3e). Wht signaling plays an essential role during embryonic muscle development and in the maintenance of skeletal muscle homeostasis ${ }^{32,33}$. Quantitative real-time polymerase chain reaction (qPCR) results confirmed the significant downregulation of the aforementioned genes (Fig. 3f). The expression of Apc showed similar pattern of change in SCs during muscle regeneration (Supplementary Fig. 2g, h). Moreover, the protein level of Apc in SCs in vivo was reduced in FoxM1-cKO mice compared with control mice (Fig. 3h, i). The cultured FoxM1-deficient SCs displayed increased total and nuclear $\beta$-catenin proteins compared with control SCs in vitro (Fig. 3g). Consistently, the protein level of $\beta$-catenin in SCs in vivo was upregulated in FoxM1deficient mice compared with control mice (Fig. 3j, k). The expression of Tgf- $\beta 2$ and Tgf- $\beta 3$ was also upregulated in FoxM1-deficient SCs in the injured condition (Supplementary Fig. 2i, j). Together, these data suggested that loss of FoxM1 resulted in activation of wnt/ $\beta$-catenin signaling in SCs.

\section{FoxM1 preserves muscle regeneration by transcriptionally regulating Apc in SCs}

To determine whether FoxM1 regulates Apc through direct transcriptional activation, we searched for the consensus FoxM1-binding site $(\mathrm{C} / \mathrm{T}) \mathrm{AAA}(\mathrm{C} / \mathrm{T}) \mathrm{AA}$ in the proximal promoter regions of Apc. A chromatin immunoprecipitation (ChIP) assay of SCs revealed the direct binding of FoxM1 to site 1 in the Apc promoter regions (Fig. 4a, b). The luciferase activity of a construct containing the wild-type (WT)-binding site showed apparent increases with FoxM1 expression. However, the construct containing the mutants did not (Fig. 4c, d). We generated mice with FoxM1 overexpression in SCs (designated as "Tg-FoxM1" mice here). The strategy efficiently promoted the overexpression of FoxM1 in SCs (Supplementary Fig. 3a-c). Inspiringly, FoxM1 overexpression significantly upregulated the expression of Apc in SCs (Fig. 4e), as well as the protein level of Apc (Fig. 4f, g). Subsequently, the total protein level of $\beta$-catenin was strikingly reduced (Fig. 4h). Loss- and gain-of-function analyses demonstrated a novel mechanism in which FoxM1 repressed wnt/ $\beta$-catenin signaling by transcriptionally regulating Apc in SCs.

To determine whether the downregulated expression of Apc hindered muscle regeneration of FoxM1-cKO mice by the Apc- $\beta$-catenin axis, the mice were administered ICG-001, a small molecule inhibitor of wnt/ $\beta$-catenin ${ }^{34}$. Treatment with ICG-001 partially rescued the regeneration capacity of FoxM1-cKO SCs, as shown by larger cross-sectional area (CSA), normal regenerative myofiber, and enhanced exercise performance (Fig. 4i-l). 


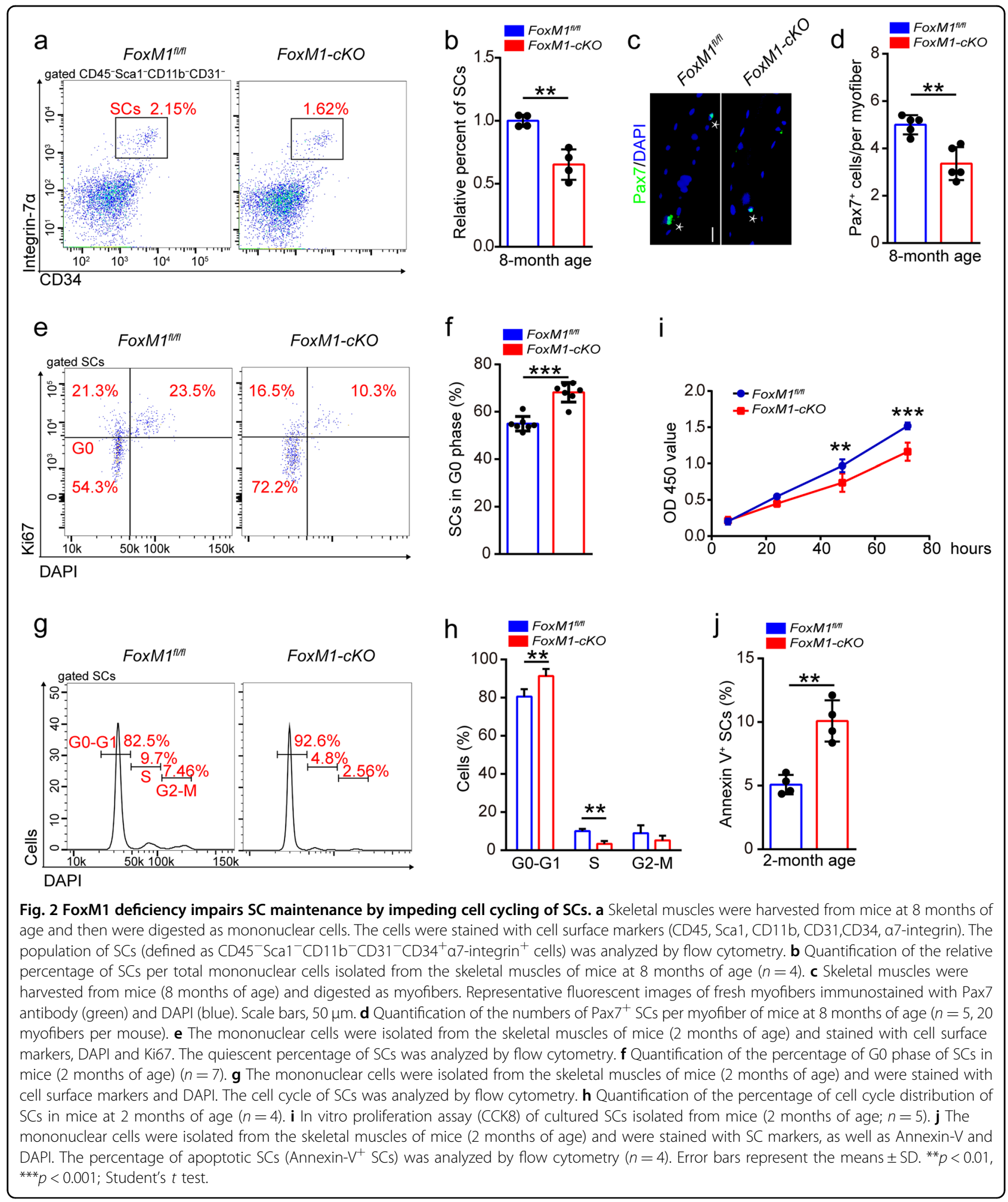

These data suggested that the regeneration defect of FoxM1-deficient SCs was partially due to the hyperactivation of wnt/ $\beta$-catenin signaling resulting from reduced Apc expression.
FoxM1 overexpression promotes muscle hypertrophy but eventually results in muscle atrophy

We observed obvious muscle hypertrophy in $T g$ FoxM1 mice compared with 2-month-old WT mice 


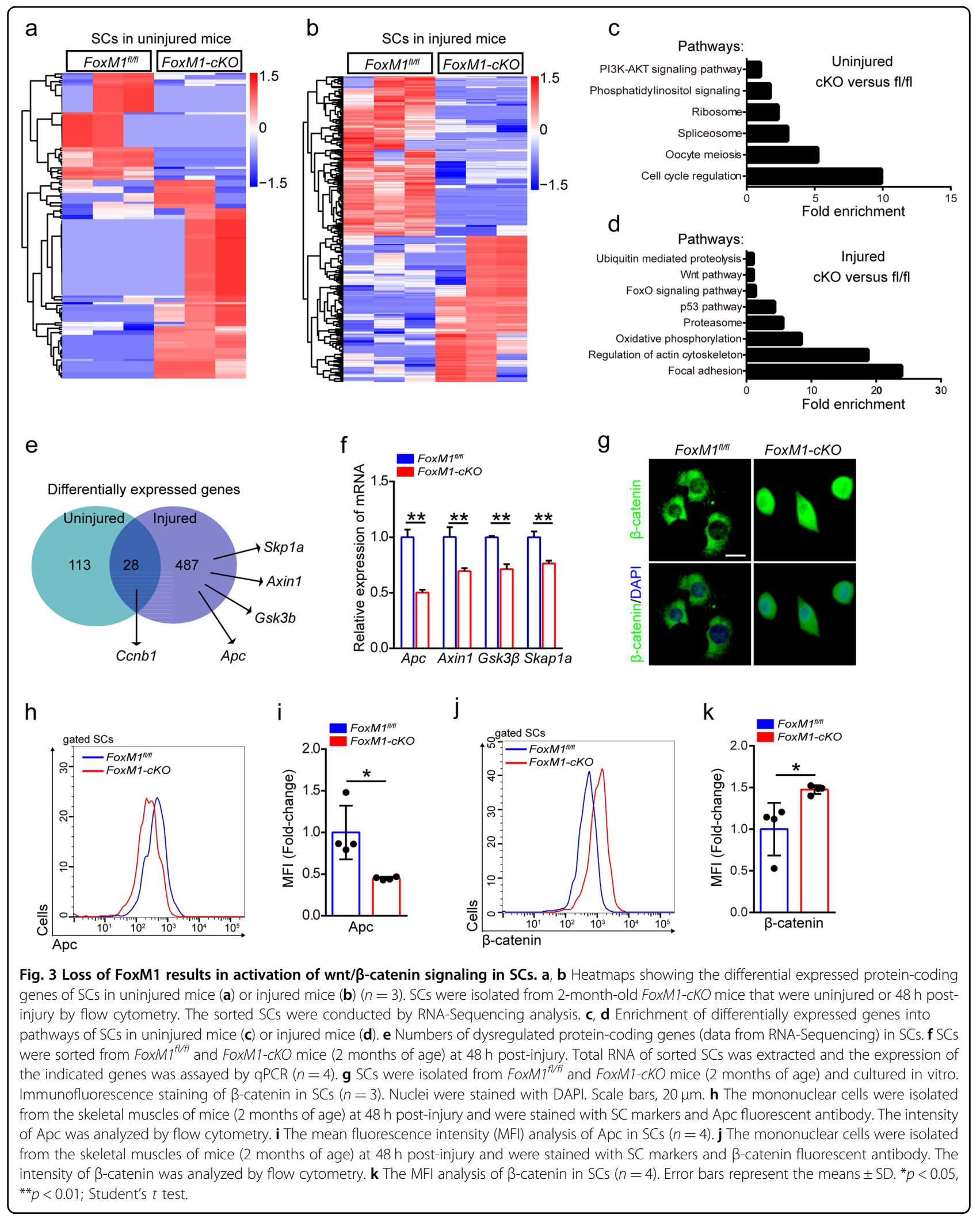




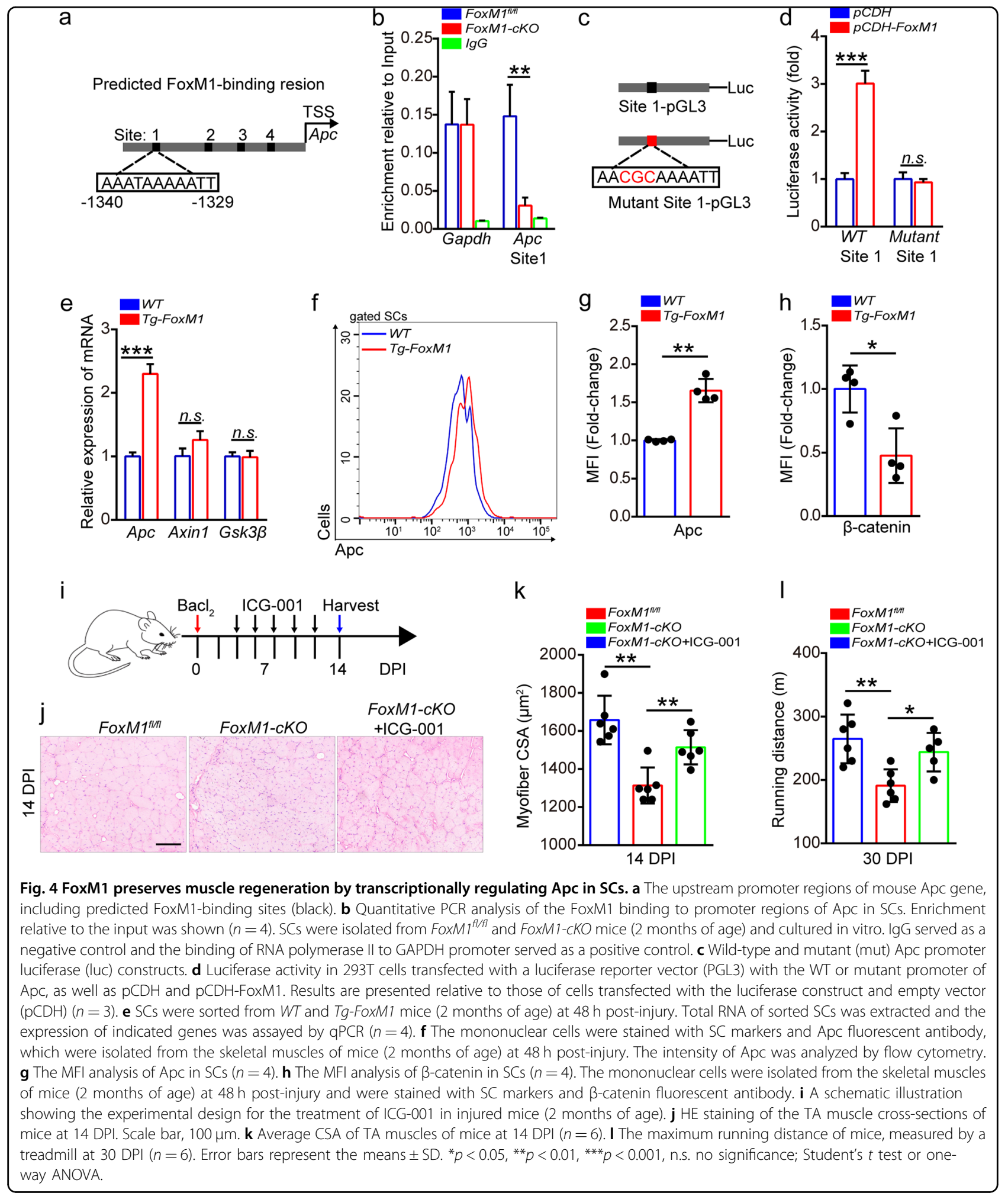

(Supplementary Fig. 3d and Fig. 5a, b). However, FoxM1 overexpression in SCs eventually resulted in striking muscle atrophy at 8 months of age (Supplementary Fig.
$3 \mathrm{e}-\mathrm{i})$. Moreover, the regenerative myofibers of 2-monthold $T g$-FoxM1 mice were significantly smaller than those in WT mice (Fig. 5c, d). These data indicated that FoxM1 

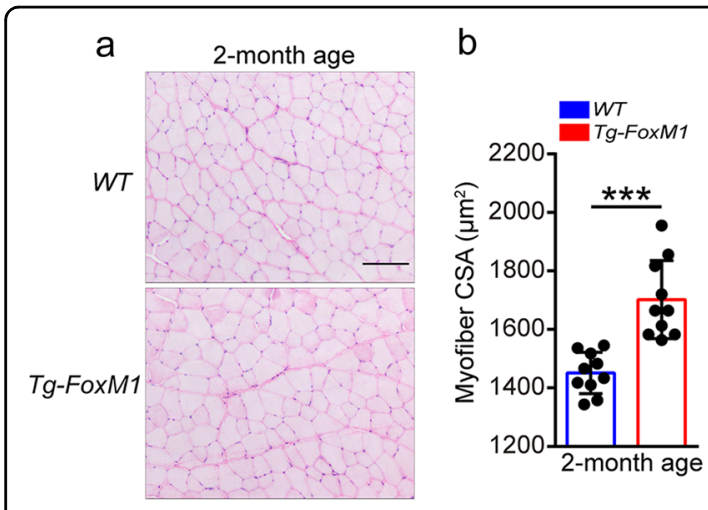

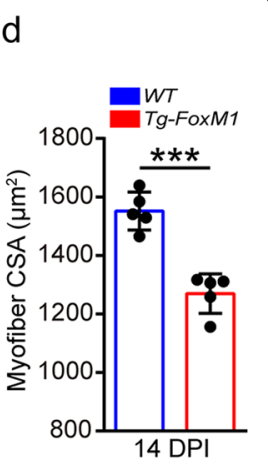

e

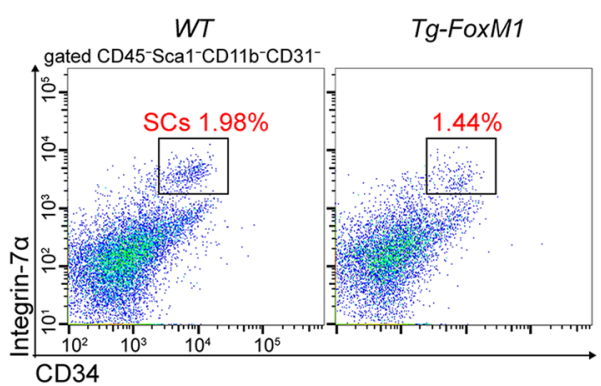

i

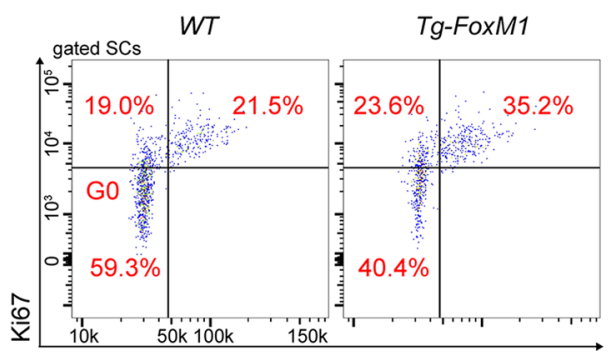

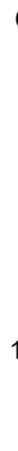

C
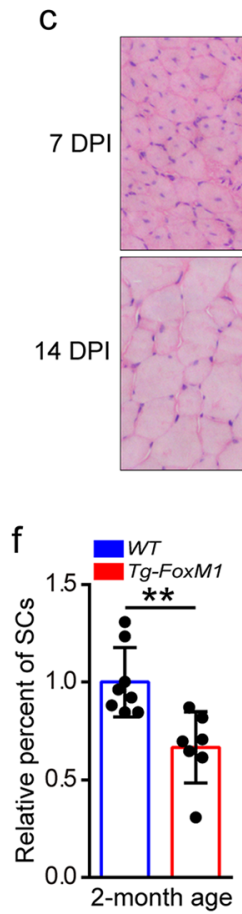

g
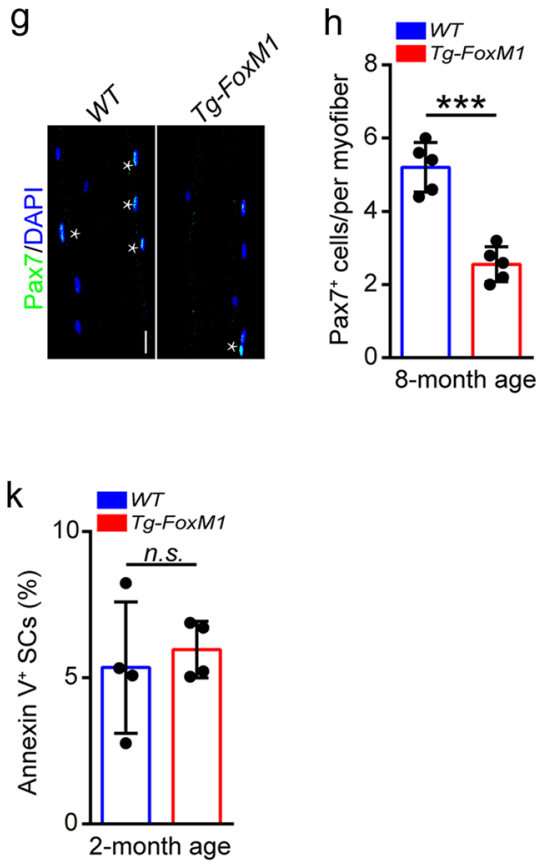

Fig. 5 FoxM1 overexpression promotes muscle hypertrophy but eventually results in muscle atrophy. a HE staining of the TA muscle crosssections in FoxM1 overexpression mice compared with control mice at 2 months of age. Scale bar, $100 \mu \mathrm{m}$. b Average CSA of TA of Tg-FoxM1 and WT mice (2 months of age) $(n=10)$. c HE staining of the TA muscle cross-sections of Tg-FoxM1 and WT mice (2 months of age) at 7 DPI and 14 DPI. Scale bar, $100 \mu \mathrm{m}$. $\mathbf{d}$ Average CSA of TA muscle of mice (2 months of age) at $14 \mathrm{DPI}(n=5)$. e The mononuclear cells were isolated from skeletal muscles of mice (2 months of age) and were stained with SC markers. The population of SCS was analyzed by flow cytometry. $\mathbf{f}$ Quantification of the relative percentage of SCs per total mononuclear cells isolated from the skeletal muscles of mice at 2 months of age $(n=8)$. $\mathbf{g}$ Skeletal muscles were harvested from mice (8 months of age) and digested as myofibers. Representative fluorescent images of fresh myofibers immunostained with Pax7 antibody (green) and DAPI (blue). Scale bars, $50 \mu \mathrm{m}$. $\mathbf{h}$ Quantification of the numbers of Pax $7^{+}$SCs per myofiber of mice at 8 months of age $(n=5,20$ myofibers per mouse). $\mathbf{i}$ The mononuclear cells were isolated from the skeletal muscles of mice (2 months of age) and were stained with cell surface markers, DAPI and Ki67. The quiescent percentage of SCs was analyzed by flow cytometry. $\mathbf{j}$ Quantification of the percentage of G0 phase in SCs of mice ( 2 months of age) $(n=6)$. $\mathbf{k}$ The mononuclear cells were isolated from the skeletal muscles of mice ( 2 months of age) and were stained with SC markers, as well as Annexin-V and DAPI. The percentage of apoptotic SCs (Annexin- $\mathrm{V}^{+}$SCs) was analyzed by flow cytometry $(n=4)$ ). Error bars represent the means \pm SD. ${ }^{*} p<0.05,{ }^{* *} p<0.01,{ }^{* * *} p<0.001$, n.s. no significance; Student's $t$ test.

overexpression in SCs promoted myogenesis at an early age but impaired muscle regeneration under stress and muscle development of mice with increasing age.

The transition of muscle hypertrophy to atrophy implied the exhaustion of SC pool in Tg-FoxM1 mice. We observed a decrease in the $\mathrm{SC}$ abundance in $\mathrm{Tg}$-FoxM1 mice compared with $W T$ mice at 2 months of age (Fig. 5e, f). The difference was more striking at 8 months of age and at 30 DPI (Supplementary Fig. 3j-l). The numbers of $\mathrm{Pax}^{+} \mathrm{SCs}$ in single fibers in Tg-FoxM1 mice were also significantly reduced compared to $W T$ mice at 8 months of age (Fig. 5g, h). These data suggested that FoxM1 
overexpression resulted in SC exhaustion, which helped explain the eventual muscle atrophy and the impaired muscle regeneration in $T g$-FoxM1 mice.

\section{FoxM1 promotes cell cycling of SCs by transcriptionally regulating Conb1}

FoxM1 overexpression promoted the entry of quiescent SCs into the cell cycle (Fig. 5i, j and Supplementary Fig. $3 \mathrm{~m}, \mathrm{n}$ ). Flow cytometric analysis revealed that FoxM1 overexpression had no obvious effect on apoptosis of SCs (Fig. 5k). FoxM1 reportedly regulates a subset of G1-G2 and G2-M transition genes. Ccnb1 showed downregulated expression in FoxM1-deficient SCs compared with control SCs (Supplementary Fig. 4a). FoxM1 overexpression significantly upregulated the expression of Ccnb1 in SCs (Supplementary Fig. 4a). The expression of Ccnb1 was strikingly induced by muscle injury (Supplementary Fig. 4b). Moreover, the protein level of Ccnb1 in SCs showed a converse change in FoxM1-cKO and Tg-FoxM1 compared to control mice in vivo (Supplementary Fig. 4c, d) and in vitro (Supplementary Fig. 4e). ChIP and luciferase reporter assays demonstrated that FoxM1 transcriptionally regulated Ccnb1 expression (Supplementary Fig. 4f-h).

Ccnb1 complexed with Cdk1 is one of the key mitotic kinases, and Cdk1/Ccnb1 governs the entry into mitosis from the G2 phase of the cell cycle ${ }^{35,36}$. We cultured SCs of FoxM1-cKO mice in vitro and enforced Ccnb1 expression in these SCs. Enforced expression of Ccnb1 partially rescued BrdU incorporation in FoxM1-deficient SCs (Supplementary Fig. 4i, j). SCs cultured in vitro were treated with RO-3306, an inhibitor of $\mathrm{Cdk} 1 / \mathrm{Ccnb1}{ }^{37}$. RO-3306 treatment in vitro abolished the increased BrdU incorporation of FoxM1-overexpressing SCs (Supplementary Fig. 4i, k). Although the injection of RO-3306 in vivo affected all muscle cells, RO-3306 treatment efficiently restored the frequency of quiescent $\mathrm{SCs}$ in $\mathrm{Tg}$ FoxM1 mice and rescued the decreased SC pool (Supplementary Fig. 5a-d). Treatment with RO-3306 had no obvious effect on the numbers of $\mathrm{Pax}^{+} \mathrm{SCs}$ but considerably recovered the decreased SC pool in Tg-FoxM1 mice (Supplementary Fig. 5e). The treatment of RO-3306 also abolished muscle hypertrophy in 2-month-old $\mathrm{Tg}$ FoxM1 mice (Supplementary Fig. 5f, g). Since Cdk1 mediates the phosphorylation of FoxM1, it is possible that the inhibition of Cdk1/Ccnb1 eventually affected the phosphorylation of FoxM1 and its transcriptional activity. Together, these data demonstrated that FoxM1 promoted cell cycling of SCs partially by transcriptionally regulating Ccnb1.

\section{Cdh1 prevents accumulation of FoxM1 protein by ubiquitylation}

The $\mathrm{APC} / \mathrm{C}$ is a multisubunit E3 ubiquitin ligase complex that regulates cyclic degradation of cell cycle regulator via Cdh1 or Cdc20 adaptor proteins ${ }^{38}$. We knocked down the expression of Cdh1 or Cdc20 by two independent short hairpin RNAs (shRNAs) in C2C12 cells. Reduction of Cdh1, but not Cdc20, resulted in increased FoxM1 protein in proliferative $\mathrm{C} 2 \mathrm{C} 12$ cells (Fig. 6a). Knockdown or knockout of FoxM1 had no obvious effect on the expression of Cdh1 and Cdc20 in $\mathrm{C} 2 \mathrm{C} 12$ or SCs (Supplementary Fig. 6a, b). Furthermore, co-immunoprecipitation assay revealed the direct interaction between FoxM1 and Cdh1, but not Cdc20, in SCs (Fig. 6b and Supplementary Fig. 6c). APC/C ${ }^{\text {Cdh1 }}$ induces the degradation of its substrates through ubiquitin-dependent proteolysis ${ }^{39}$. As expected, knockdown of Cdh1 reduced poly-ubiquitylation of FoxM1 in C2C12 cells (Fig. 6c, d). These data suggested that APC/ $\mathrm{C}^{\mathrm{Cdh} 1}$ promoted FoxM1 degradation through the ubiquitin-proteasome pathway in $\mathrm{C} 2 \mathrm{C} 12$ cells in vitro.

We generated the conditional knockout mouse $C d h f^{f l}$ ${ }^{f l}$ Pax7-Cre (designated as Cdh1-cKO) and its control $\left(C d h 1^{f l / f l}\right)$ (Supplementary Fig. 6e, f). The FoxM1 protein level in Cdh1-cKO SCs was increased compared to Cdh $f^{l /}$ ${ }^{f l}$ SCs in vitro (Fig. 6e). The mRNA and protein levels of Cdh1 showed peaks at 7 DPI (Supplementary Fig. 6d). The protein level of FoxM1 in SCs was strikingly elevated in Cdh1-cKO mice (Fig. 6f, g). Together, these data suggested that Cdh1 prevented accumulation of FoxM1 protein by ubiquitylation.

\section{Loss of Cdh1 results in the loss of SC pool}

Knockout of Cdh1 evidently decreased the percentage of quiescent SCs (Fig. 7a, b) and depleted the SC pool at 2 months of age (Fig. 7d, e). Moreover, we detected muscle hypertrophy in Cdh1-cKO mice compared to $C d h 1^{f l f l}$ mice at the same age (Fig. 7c). We generated $C d h f^{f l / f l}$, FoxMP $1^{f l+}$, and Pax7-Cre mice to test whether haploinsufficiency of FoxM1 could rescue the exhaustion of SCs in Cdh1-cKO mice. Deletion of Cdh1 resulted in a remarkable reduction of SCs at $30 \mathrm{DPI}$, while haploinsufficiency of FoxM1 partially restored the quantity of SCs (Fig. 7f). Consistently, serial injuries resulted in a severe regeneration defect in Cdh1-cKO mice, but haploinsufficiency of FoxM1 improved the muscle regeneration of Cdh1-deficient mice (Fig. 7g, h). Together, these data suggested that Cdh1 prevented FoxM1 accumulation to maintain muscle homeostasis.

\section{Discussion}

FoxM1 is a well-established regulatory transcriptional factor for cell cycle progression ${ }^{40,41}$. Recently, increasing evidence has suggested the pleiotropic role of FoxM1 in stem cells ${ }^{19,20}$. In this study, we identified previously unrecognized roles of FoxM1 in muscle homeostasis and SC maintenance. We found that FoxM1 promoted cell cycling of SCs partially by transcriptionally regulating 


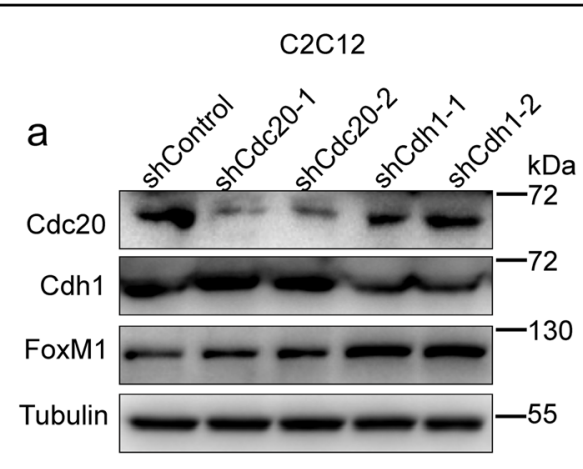

C

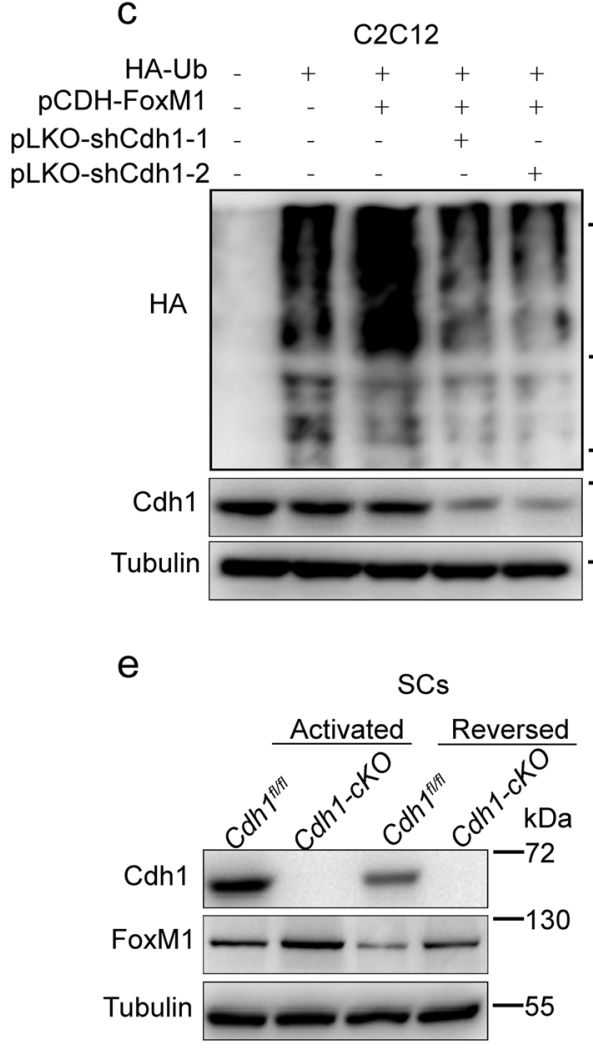

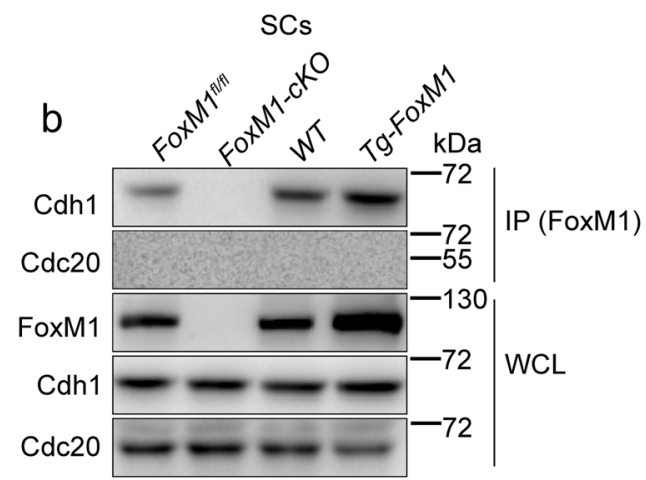

d

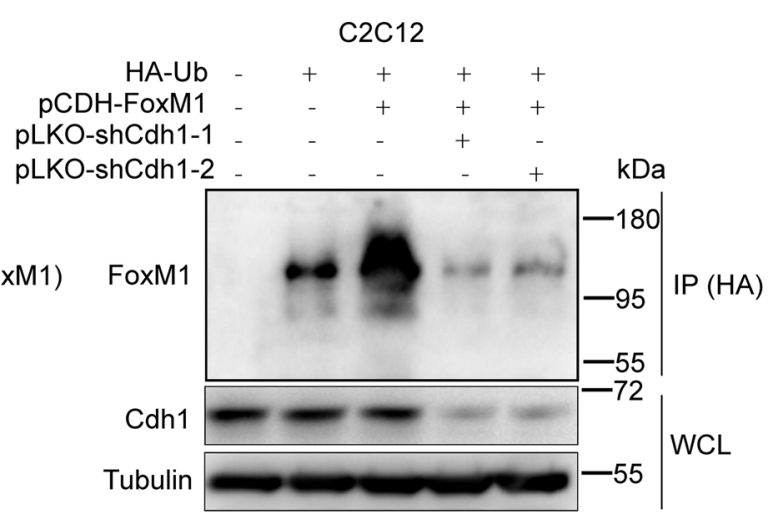

WCL

f

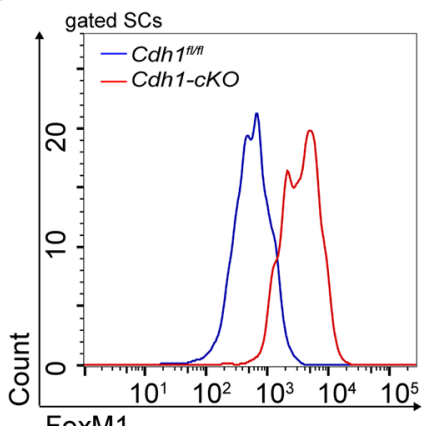

Fig. 6 Cdh1 prevents accumulation of FoxM1 protein by ubiquitylation. a Cdc20 and Cdh1 were knocked down in proliferative C2C12 using independent shRNAs (pLKO construct). Levels of Cdc20, Cdh1, FoxM1, and Tubulin (loading control) were determined by western blot analysis $(n=3)$. $\mathbf{b}$ SCs were isolated from FoxM1 deletion or overexpression mice and were cultured in vitro. Cdh1 proteins were immunoprecipitated from the cell lysates and analyzed by western blot $(n=3)$. WCL whole-cell lysates. $\mathbf{c}$, d HA-ubiquitin (HA-Ub, ubiquitin expression construct with HA tag) plasmid was transfected with the indicated plasmids (FoxM1 overexpression plasmid, pCDH-FoxM1; Cdh1 knockdown plasmid, pLKO-shCdh1-1, pLKO-shCdh1-2) in proliferative C2C12 cells; $8 \mathrm{~h}$ after $10 \mu \mathrm{M}$ MG132 treatment, HA-Ubiquitin (c) or FoxM1 (d) proteins were immunoprecipitated from cell lysates and analyzed by western blot $(n=4)$. WCL whole-cell lysates. e SCs were isolated from $C d h 1^{f / f l}$ and $C d h 1^{f / f / P} P a x 7-C r e ~(c a l l e d ~ C d h 1-C K O$ here) and were cultured in growth medium (activated state) or serum-free medium (reversed state) in vitro. Whole-cell lysates were subjected to western blot analysis using the indicated antibodies $(n=3)$. f The mononuclear cells were isolated from skeletal muscles of mice $(2$ months of age) and were stained with SC markers and FoxM1 fluorescent antibody. The intensity of FoxM1 was analyzed by flow cytometry. $\mathbf{g}$ The MFI analysis of FoxM1 in SCs $(n=3-4)$. Error bars represent the means \pm SD. ${ }^{*} p<0.05$; Student's $t$ test. 

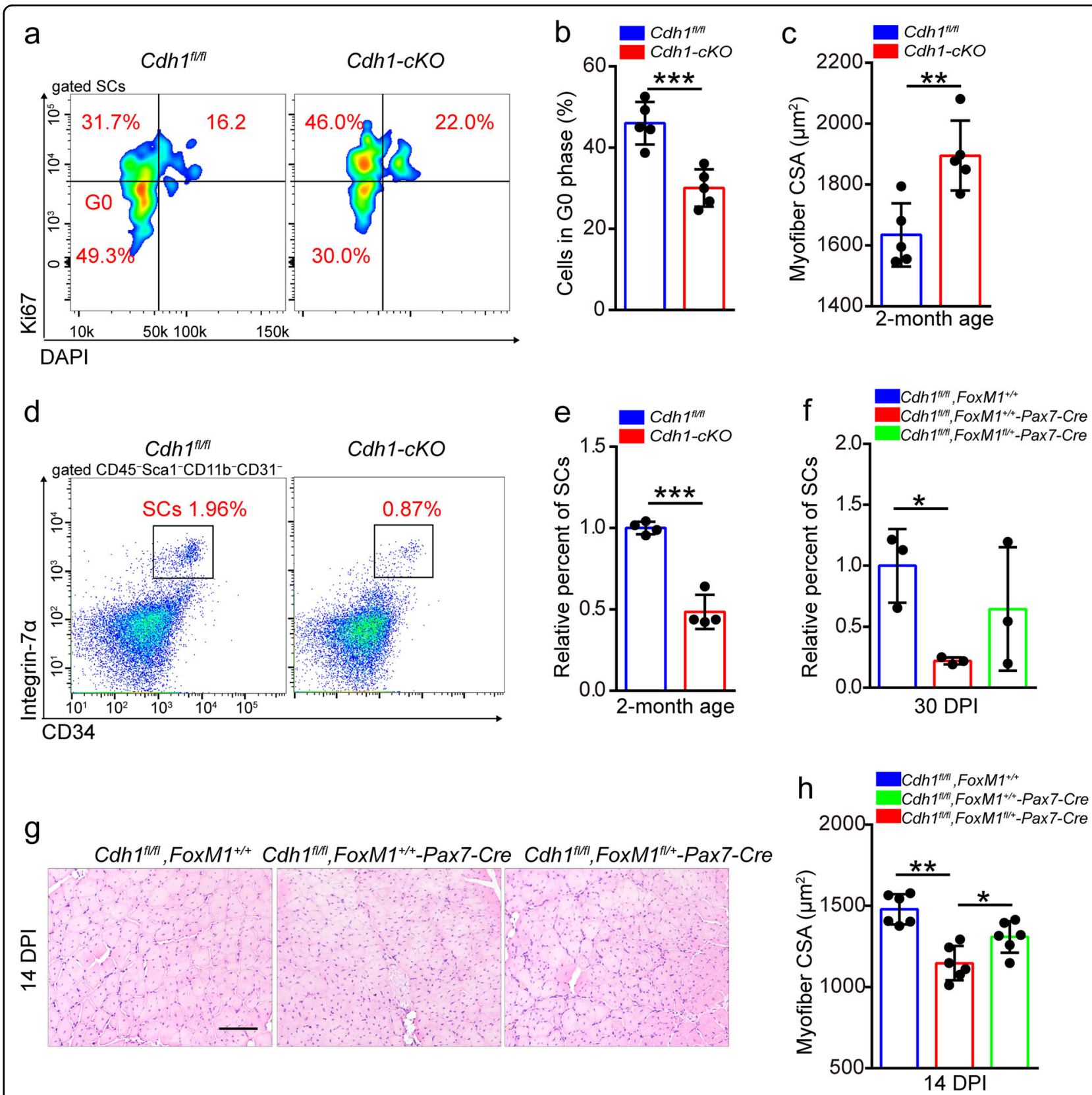

Fig. 7 Loss of Cdh1 results in the loss of SC pool. a The mononuclear cells were isolated from the skeletal muscles of mice ( 2 months of age) and were stained with cell surface markers, DAPI and Ki67. The quiescent percentage of SCs was analyzed by flow cytometry. $\mathbf{b}$ Quantification of the percentage of GO phase in SCs of mice ( 2 months of age) $(n=5)$. c Average CSA of TA in Cdh $1^{f / t}$ and Cdh 1 -cKO mice $(2$ months of age) $(n=5)$. $\mathbf{d}$ The mononuclear cells were isolated from skeletal muscles of mice (2 months of age), and were stained with SC markers. The population of SCs was analyzed by flow cytometry. e Quantification of the relative percentage of SCs per total mononuclear cells isolated from the skeletal muscles of mice at 2 months of age $(n=4)$. $\mathbf{f}$ Quantification of the relative percentage of SCs per total mononuclear cells isolated from the skeletal muscles of mice (2 months of age) at $30 \mathrm{DPI}(n=3)$. $\mathbf{g}$ Mice were induced with serial muscle injuries by $\mathrm{BaCl}_{2}$ injection. HE staining of the TA muscle cross-sections of mice (2 months of age) at 14 DPI of the second injury. Scale bar, $100 \mu \mathrm{m}$. $\mathbf{h}$ Average CSA of TA muscles of mice ( 2 months of age) at 14 DPI of the second injury $(n=6)$. Error bars represent the means \pm SD. ${ }^{*} p<0.05,{ }^{* *} p<0.01,{ }^{* *} p<0.001$; Student's $t$ test or one-way ANOVA.

Ccnb1. FoxM1 also preserved regenerative potential of SCs by transcriptionally regulating Apc to inhibit wnt/ $\beta$-catenin signaling. The degradation of FoxM1protein is regulated by $\mathrm{APC} / \mathrm{C}^{\mathrm{Cdh} 1}$ via ubiquitylation.
FoxM1 acts as a cell cycle regulator and induces cell proliferation ${ }^{42-44}$. In this study, we found that loss of FoxM1 in SCs resulted in decreased SC pool with age, and the percentage of quiescent SCs considerably reduced. 
However, overexpression of FoxM1 evidently promoted cell cycle entry of SCs and enhanced proliferative capacity of SCs. It is possible that the quiescence exit and the increased proliferation of $\mathrm{SCs}$ in $\mathrm{Tg}$-FoxM1 mice in combination results in SC exhaustion with age or under stress. Muscle development relies on proliferation and differentiation potential of SCs. We found that loss of FoxM1 had no obvious effect on SC differentiation. Thus the eventual muscle atrophy observed in FoxM1-cKO and $T g$-FoxM1 mice could be attributed to the altered cell cycle of SCs and the reduced SC pool.

FoxM1 has been reported to regulate a subset of G1-G2 and $\mathrm{G} 2-\mathrm{M}$ transition genes in stage- and contextdependent manners ${ }^{42-44}$. Ccnb1 is a transcriptional target of FoxM1 in human hepatocellular carcinoma cells ${ }^{45}$ and human hTERT RPE-1 cells ${ }^{46}$. Ccnb1 binds to its partner Cdk1, and $\mathrm{Cdk} 1 / \mathrm{Ccnb} 1$ is crucial for $\mathrm{G} 2-\mathrm{M}$ transition of the cell cycle ${ }^{47}$. Cdk1 can directly phosphorylate FoxM1 to increase its transcriptional activity $^{46,48}$. Presently, FoxM1 directly regulated Ccnb1 expression by binding to its proximal promoter region. Inhibition of $\mathrm{Cdk} 1 / \mathrm{Ccnb} 1$ partially rescued the loss of quiescent SCs in FoxM1-overexpressing mice. Thus we consolidated the reported mechanisms of FoxM1 transcriptionally regulating Ccnb1. We also found that ribosome-related genes were downregulated in FoxM1deficient SCs, which helped explain the phenomenon that the enforced expression of Ccnb1 could not completely recover proliferation capacity of FoxM1-deficient SCs. Our data suggest that FoxM1 maintains the SC pool partially by transcriptionally regulating Ccnb1. Whether FoxM1 promotes the proliferation of SCs by ribosome biogenesis requires further studies.

Besides the effect of FoxM1 on SC cell cycling, we found that loss of FoxM1 severely impaired the regeneration potential of SCs. FoxM1-cKO mice showed largely unrepaired muscle regions compared to FoxM1 $1^{f l f l}$ mice, which could not be simply attributed to the altered cell cycle of SCs. Through RNA-Sequencing, we found that knockdown of FoxM1 resulted in downregulation of genes related to the wnt pathway, including Apc, Axin1, and Gsk3 $\beta$. Importantly, muscle regeneration required inhibited wnt $/ \beta$-catenin signaling and Apc interaction with Axin, Cki, and Gsk3 $\beta$ to form a cytoplasmic destruction complex to phosphorylate $\beta$-catenin, leading to destruction of $\beta$-catenin via the ubiquitylation pathway $^{27}$. The loss of Apc in SCs does not affect skeletal muscle tissue integrity but results in severe muscle regeneration defects ${ }^{33}$. $\beta$-Catenin constitutively active mice are not affected with respect to the number of SCs in the homeostatic condition. However, these mice display muscle regeneration defects with aberrant myofiber morphology and atrophy, which is mainly attributed to hyperactive Tgf- $\beta$ signaling ${ }^{28}$. The phenotype of muscle regeneration in FoxM1-cKO mice was similar to that in Apc-deficient and $\beta$-catenin constitutively active mice. Furthermore, the protein level of $\beta$-catenin in FoxM1deficient SCs was considerably increased following injury, raising a possibility that FoxM1 can inhibit wnt/ $\beta$-catenin signaling to preserve regenerative potential of SCs.

The crosstalk of FoxM1 and wnt/Apc/ $\beta$-catenin signaling is complicated. It has been reported that Gsk3 phosphorylates FoxM1 on serine 474, which induces FoxM1 ubiquitylation and subsequent degradation in LN229 and U87 cells ${ }^{25}$. Wnt3a increases the level and nuclear translocation of FoxM1, which binds directly to $\beta$-catenin and enhances $\beta$-catenin nuclear localization in $293 \mathrm{~T}$ cells ${ }^{26}$. Presently, FoxM1 transcriptionally regulated the expression of Apc to restrict the activation of $\beta$-catenin signaling in SCs under stress. We speculate that the differing observations of our study may be attributed to the differences in cell types. Inhibition of wnt/ $\beta$-catenin signaling by ICG-001 efficiently rescued the aberrant myofiber morphology and atrophy in FoxM1-cKO mice after injury. These findings expand our insights into the interwoven intercommunication between FoxM1 and wnt $/ A p c / \beta$-catenin signaling and reveal the protective function of FoxM1 in muscle regeneration. This may be an adaptive strategy for SCs upon injury. Whether the FoxM1-Apc- $\beta$-catenin axis exists in other stem cells is unclear and warrants further investigation.

FoxM1 was required for SC proliferation and muscle regeneration, but excessive FoxM1 resulted in SC exhaustion. The expression of FoxM1 in SCs during muscle regeneration also showed a process of upregulation to downregulation. The protein level of FoxM1 was evidently increased in activated $\mathrm{C} 2 \mathrm{C} 12$ compared to quiescent cells ${ }^{21}$ and notably decreased when returning to quiescent state (for $\mathrm{C} 2 \mathrm{C} 12$ and SCs). Thus a regulatory network may precisely control the expression of FoxM1 at the transcriptional and epigenetic level. Proteolysis by the ubiquitin-proteasome system is a major mechanism involved in myofibrillar protein degradation. FoxM1 interacts with the complex of E3 ligase APC/C and its adaptor, Cdh1. Cdh1 stimulated degradation of FoxM1 protein in HeLa ${ }^{49}$, U2OS ${ }^{17}$, and MCF-7 cells ${ }^{50}$. Moreover, Fbxl2 (a component of Skp-Cullin-F box ubiquitin E3 ligase $)^{51}$ and VprBP (a component of cullin 4-based E3 ubiquitin ligase) ${ }^{52}$ have been reported to regulate the degradation of FoxM1 in different cells. Here we discovered that FoxM1 directly interacted with Cdh1, rather than the adaptor Cdc20. Knockdown or knockout of Cdh1 resulted in the accumulation of FoxM1 protein in SCs. Cdh1 was required for FoxM1 ubiquitylation and subsequent degradation. The findings expand our knowledge of the relationship between Cdh1 and FoxM1 in stem cells and demonstrate that 
$\mathrm{APC} / \mathrm{C}^{\mathrm{Cdh} 1}$ mediates ubiquitylation and degradation of FoxM1 protein in SCs.

Cdh1 has been implicated in regulating cell cycle entry. Presently, deletion of Cdh1 resulted in decreased quiescent SCs and SC exhaustion. This phenotype is similar to that of FoxM1-deficient SCs. The haploinsufficiency of FoxM1 partially recovered the maintenance of SCs after injury and rescued the muscle regeneration of Cdh1-cKO mice, implying that FoxM1 was one of the key downstream targets of Cdh1 in vivo. The accumulation of ubiquitinated protein increased at $3 \mathrm{DPI}$, indicating that muscle regeneration is associated with the activation of machinery involved in protein degradation ${ }^{12}$. We observed that knockout of Cdh1 in SCs resulted in impaired muscle regeneration, indicating the important function of ubiquitin-proteasome system in muscle regeneration. Whether Cdh1 regulates SCs maintenance and muscle development with age requires further investigation.

In summary, the data presented here demonstrate that FoxM1 was critical for muscle development and regeneration. FoxM1 regulated cell cycle entry of SCs to meet the needs of myogenesis and muscle development, partially via Ccnb1. Upon injury, FoxM1 transcriptionally regulated Apc expression to repress wnt/ $\beta$-catenin signaling. The ubiquitylation and degradation of FoxM1 protein in $\mathrm{SCs}$ was promoted by $\mathrm{APC} / \mathrm{C}^{\mathrm{Cdh} 1}$. These findings uncover an Cdh1-FoxM1-Apc axis that regulates muscle development and regeneration. Whether this axis is associated with muscle diseases and whether it could be potential intervention targets warrants further investigations.

\section{Methods}

\section{Mice and drug treatment}

The mice used had a C57BL/6J background. The FoxM1 $1^{f l+}$ mice carry a FoxM1 locus in which exons 4-7 were flanked by 2 loxp sites, while $C d h 1^{f l /+}$ mice carry a Cdh1 locus in which exons 2-14 were flanked by 2 loxp

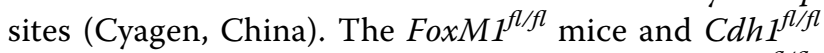
was crossed with Pax7-Cre mice to generate FoxM1 $1^{f l f l_{-}}$ Pax7-Cre SC-specific knockout mice (FoxM1-cKO) and $C d h 1^{f l / f l}$-Pax7-Cre SC-specific knockout mice (Cdh1$c K O$ ). For SC-specific overexpression of FoxM1, we generated a bacterial artificial chromosome (BAC) transgenic mouse lines, in which a "FoxM1 cdsrBGpA" cassette was inserted into the BAC RP23267J16 following the start codon (ATG) in exon 1 of Pax7 gene (Tg-FoxM1). All compound genetically engineered mice were a result of breeding of the above mice followed by appropriate PCR-based genotyping with specific primers (Table S1). For drug treatments, ICG-001 (20 mg/kg, 3 injections/week, Selleck) and RO3306 (4 mg/kg, 4 injections/week, Selleck) were intraperitoneally injected into mice for 2 and 5 weeks, respectively. All the mice experiments were approved by the Animal Committee of the Institute of Zoology, Third Military Medical University.

\section{Histology and morphometric analysis}

TA muscles harvested and were fixed for $12 \mathrm{~h}$ using $4 \%$ paraformaldehyde and subsequently transferred to $20 \%$ sucrose overnight for dehydration. For the assessment of muscle morphology, 10- $\mu$ m-thick transverse sections of TA muscle were subjected to hematoxylin and eosin staining. For quantitative analysis, the CSA was performed using the AxioVision software (Zeiss).

\section{Skeletal muscle injury}

To induce injury, $50 \mu \mathrm{l} 1.2 \% \mathrm{BaCl}_{2}$ in phosphatebuffered saline (PBS) or PBS alone was injected into and along the length of TA and gastrocnemius muscles. The hindlimb muscles or TA muscles were harvested 2-30 days after injury depending on the experiments.

\section{Treadmill test}

Mice were acclimated to moderate treadmill running $(10 \mathrm{~m} / \mathrm{min}$ for $5 \mathrm{~min}$ daily) on a treadmill (ZH-PT, Zheng Hua) for 3 consecutive days before the test. Then mice ran on the treadmill at $10 \mathrm{~m} / \mathrm{min}$ for $5 \mathrm{~min}$, and the speed was increased from 10 to $16 \mathrm{~m} / \mathrm{min}$ and then maintained constant until exhaustion. Running time and running distance were measured.

\section{Isolation of single myofibers}

Single myofibers were isolated from EDL muscles and digested in Dulbecco's modified Eagle's medium (DMEM; catalog number: SH30022.01, Hyclone, GE, USA) with $0.2 \%$ collagenase (Sigma-Aldrich, USA) at $37^{\circ} \mathrm{C}$ for $90 \mathrm{~min}$. Fibers were liberated by trituration in DMEM medium with Pasteur pipettes.

\section{SC isolation and culture}

To culture SCs in vitro, we obtained SCs using single myofiber cultures. Briefly, single fibers were placed in Matrigel-coated dishes (BD Bioscience, USA) in fiber medium consisting of DMEM (Hyclone) with $20 \%$ fetal bovine serum (Hyclone), 1\% penicillin/streptomycin (Hyclone), and 1\% Chick embryo extract (US biological, USA) at $37^{\circ} \mathrm{C}$ with $5 \% \mathrm{CO}_{2}$. SCs migrated off the myofibers in 3-4 days. The correct enrichment of SCs were validated by fluorescence staining of Pax7. To analyze the growth of SCs, isolated SCs were cultured in fiber medium. For inducing the reversed state of SCs, cells were cultured in serum-free DMEM for $72 \mathrm{~h}$. For differentiation, SCs were cultured in DMEM containing 2\% horse serum (Gibco, Thermo Fisher, USA) on Matrigel. For the inhibition of Cdk1/Ccnb1, SCs were treated with $0.5 \mu \mathrm{M}$ RO-3306 (Selleck) for $24 \mathrm{~h}$ before analysis. 


\section{Flow cytometry}

SC isolation and purification was performed according to the established methods. Briefly, TA muscle of mice was subjected to $0.2 \%$ collagenase (Sigma) for $90 \mathrm{~min}$ and then $0.2 \%$ dispase (Sigma) for $30 \mathrm{~min}$. The cell suspension was filtered through a 70- $\mu \mathrm{m}$ nylon filter (Falcon), and mononuclear cells were collected and subjected to fluorescence-activated cell sorter (FACS; BD FACSAriaII) using immunostaining with the following: biotin antimouse CD45 (Biolegend, 103104), biotin anti-mouse/ human CD11b (Biolegend, 101204), biotin anti-mouse CD31 (Biolegend, 102404), biotin anti-mouse Sca1 (Biolegend, 108103), streptavidin-APC/Cy7 (Biolegend, 405208), CD34-Alexa Fluor 647 (Biolegend, 152205), and Integrin $\alpha 7$-FITC (eBioscience, 11-5867-42). For the intracellular staining, the SCs were first stained with the surface marker and then subjected to Fixation/Permeabilization Kit (BD) according to the manufacturer's instruction. Then the cells were stained with anti-Ki67-PE (Biolegend, 652404) or anti-Cyclin B1-PE (Biolegend, 647903) or stained with anti-Apc (Beyotime, AF2113) or anti-Cdh1 (Proteintech, 16368-1-AP) and incubated with anti-rabbit-FITC (Biolegend, A120-101F).

For BrdU incorporation analysis, SCs cultured in vitro were incubated with $10 \mu \mathrm{M}$ BrdU (Sigma) for $30 \mathrm{~min}$, washed with PBS, fixed with $70 \%$ absolute ethyl alcohol for $6 \mathrm{~h}$, permeabilized with $0.1 \%$ Triton X-100 for $10 \mathrm{~min}$, incubated with $2 \mathrm{M} \mathrm{HCl}$ for $30 \mathrm{~min}$, and blocked with $2 \%$ horse serum for $1 \mathrm{~h}$. Then cells were stained with antiBrdU-FITC (Biolegend, 364104) for $30 \mathrm{~min}$ and analyzed by flow cytometry.

\section{Immuofluorescence}

Myofibers or SCs were fixed with 4\% paraformaldehyde for $30 \mathrm{~min}$ at room temperature, washed, and then incubated in $0.1 \%$ Trixon X-100 for $10 \mathrm{~min}$. Then samples were blocked with $2 \%$ horse serum for $1 \mathrm{~h}$ and incubated with primary antibodies at $4{ }^{\circ} \mathrm{C}$ overnight. The following antibodies were used: Pax7 (Invitrogen, PA1-117, 1:100), MyHC (R\&D, MAB4470, 1:50), and $\beta$-catenin (Proteintech, 51067-2-AP, 1:200). After the primary antibody incubation, myofibers or SCs were incubated with secondary antibodies conjugated with Alexa Fluor 488 or 647 (Beyotime, 1:500). Finally, the nuclei were staining by 4,6diamidino-2-phenylindole $(5 \mu \mathrm{g} / \mathrm{ml}$, Sigma) for $10 \mathrm{~min}$, and the fluorescent pictures were captured by confocal microscopy (Olympus).

\section{Cell proliferation assay}

To assess the altered cell phenotypes, we seeded the SCs in 96-well plates with 2000 cells per well in triplicate. At 24,48 , and $72 \mathrm{~h}$ post culture, cell proliferation was assessed using the Cell Counting Kit-8 Kit (Beyotime) according to the manufacturer's instruction.

\section{Library preparation and RNA-Sequencing}

About $800 \mathrm{SCs}$ from mice (homeostatic condition or $48 \mathrm{~h}$-post injury) were isolated by FACS, and the mRNA library was constructed using the QIAseq FX Single Cell RNA Library Kit (QIAGEN) according to the manufacturer's instruction. Libraries were sequenced by the Illumina HiSeq 2000 platform as 150-bp pair-ended reads. Reads were aligned using bowtie v0.12.9. FPKM estimation was performed with Cufflinks v2.1.1, aligned reads were counted with HTSeq, and differential expression analysis was performed with DESeq2. Differentially expressed genes were selected using a cut-off at a $p$ value of $<0.05$ (false discovery rate adjusted for multiple testing).

\section{RNA extraction and qPCR}

Total cellular RNA was isolated from 3000 SCs immediately after FACS sorting using the Total RNA Isolation Kit (Thermo Fisher) according to the manufacturer's instructions. cDNA was reverse transcribed using the PrimeScript RT Reagent Kit (Takara) and subjected to real-time PCR with SYBR Green Supermix (Bio-Rad) in an iCycler iQ Real Time PCR Detection System (Bio-rad). All primers are listed in Table S1. All samples were run in triplicate. $\beta$-Actin (Actinb) was used as an internal control for mRNA.

\section{Lentiviral constructs and packaging}

To generate the vectors for the expression of FoxM1, CDC20, and CDH1-specific shRNA, we designed the sequence of shRNAs and cloned shRNAs into the vector pLKO.1-puro (primer sequences, Table S1). For generation of the vectors of expression of FoxM1, we amplified transcriptional regions by reverse transcription-PCR and cloned the regions into pCDH-puro (primer sequences, Table S1). The HA-Ubiquitin plasmid was a gift from Dr. Zhigang Li. For plasmid transfection, C2C12 or 293T cells were transfected with the indicated plasmids using polyetherimide (Sigma-Aldrich, USA). For lentivirus production, 293T cells were transfected with the helper plasmid pSPAX2 and pMD2.G. The medium was replaced with fresh medium at $10 \mathrm{~h}$ after transfection. The culture supernatants were collected at $48 \mathrm{~h}$ after transfection and filtered by $0.22-\mu \mathrm{m}$ membrane. Virus was stored at $-80^{\circ} \mathrm{C}$ until use. For lentiviral infection of cells, lentiviral stock was added to $\mathrm{C} 2 \mathrm{C} 12$ or SCs with polybrene $(8 \mu \mathrm{g} / \mathrm{ml}$, SigmaAldrich, USA). After incubation overnight, supernatants was removed, and cells were washed with PBS and then resuspended in fresh medium with or without puromycin ( $3 \mu \mathrm{g} / \mathrm{ml}$, Sigma-Aldrich) to screen stable cell lines.

\section{Immunoprecipitation and western blot}

Cells were prepared using RIPA buffer (Beyotime) containing protease inhibitor mixture (Beyotime). Immnoprecipitates or total cell lysates were analyzed by western blot according to standard procedures. The antibodies used in 
this study were against: Cdc20 (Proteintech, 10252-1-AP, 1:1000), Cdh1 (Santa, sc-56312 1:1000), FoxM1 (Proteintech, 13147-1-AP, 1:1000), Cyclin B1 (CST, 4138T, 1:1000), Cyclin B2 (Beyotime, AF2509, 1:1000), Apc (Beyotime, AF2113, 1:1000), $\beta$-catenin (Proteintech, 510672-AP, 1:1000), HA (Proteintech, 66006-2-Ig, 1:10,000), and Tubulin (Beyotime, AF0001, 1:1000).

\section{ChIP assay}

SCs were harvested and used for ChIP assays according to the manufacturer's instruction of the EZ-ChIP Chromatin Immunoprecipitation Kit (Millipore). In brief, the cells were fixed with $1 \%$ formaldehyde for $10 \mathrm{~min}$, and the fixation reaction was quenched with glycine to a final concentration of $125 \mathrm{mM}$. The cells were lysed and sonicated until the desired lengths were achieved $(100-500 \mathrm{bp})$. Then $5 \mu \mathrm{g}$ of anti-FoxM1 (Proteintech) or control IgG were used for immunoprecipitation. After elution of DNA from the precipitated immunocomplexes, PCR or qPCR were performed with the specific primers (Table S1).

\section{Luciferase reporter assay}

The corresponding genomic regions that included the putative binding sites was amplified by PCR from WT C57BL/6 mouse genomic DNA (primer sequences, Table S1), then were subsequently cloned into a pGL3-basic luciferase reporter vector. The vectors with mutant-binding sites were generated by site-directed mutagenesis with 3 bases' mutation (primer sequences, Table S1). The vectors were transfected into 293T cells through the use of the polyetherimide, together with either $\mathrm{pCDH}$ or $\mathrm{pCDH}$ FoxM1, as well as phRL-SV40 vector as an internal control. Cells were collected at $36 \mathrm{~h}$ after transfection, and both luciferase activities were assessed with the Dual Luciferase Reporter Gene Assay Kit according to the manufacturer's instructions (Beyotime Biotechnology, China).

\section{Statistical analysis}

All experiments included at least three biological replicates. Comparisons between two groups were analyzed using two-tailed Student's $t$ test. Differences among more than two groups were analyzed using one-way analysis of variance followed by Tukey-Kramer post hoc tests. Values of $p<0.05$ were considered statistically significant. All data are means \pm SD.

\section{Accession numbers}

All sequencing data are deposited in the NCBI GEO database under the accession number: 135486.

\section{Acknowledgements}

We thank Professor You-Yang Zhao (University of Illinois College of Medicine) for providing the FoxM $7^{f / f l}$ mice. This work was supported by grants from National Key R\&D Program of China (2017YFA0106700) and National Science Foundation of China $(81670096,31401253$, and 81700135$)$ and sponsored by
Natural Science Foundation of Chongqing, China (CSTC2016JCYJA0354), Major Technological Innovation Plan of Southwest Hospital (SWH2016ZDCX1010), Army Major Scientific Research Projects (AWS17J007), and Military Medical Innovation Program (SWH2018LJ-07 and SWH2018QNKJ-28).

\section{Author details}

'Department of Hematology, Southwest Hospital, Third Military Medical University (Army Medical University), 400038 Chongqing, China. ${ }^{2}$ Medical Research Center, Southwest Hospital, Third Military Medical University (Army Medical University), 400038 Chongqing, China. ${ }^{3}$ Key Laboratory of Biorheological Science and Technology, Ministry of Education, College of Bioengineering, Chongqing University, 400044 Chongqing, China.

${ }^{4}$ Department of Medicine, College of Medicine, University of Illinois at Chicago, Chicago, IL 60612, USA

Conflict of interest

The authors declare that they have no conflict of interest.

\section{Publisher's note}

Springer Nature remains neutral with regard to jurisdictional claims in published maps and institutional affiliations.

Supplementary Information accompanies this paper at (https://doi.org/ 10.1038/s41419-020-2375-6).

Received: 18 November 2019 Revised: 15 February 2020 Accepted: 18 February 2020

Published online: 09 March 2020

\section{References}

1. Le Grand, F. \& Rudnicki, M. A. Skeletal muscle satellite cells and adult myogenesis. Curr. Opin. Cell Biol. 19, 628-633 (2007).

2. Bentzinger, C. F., Wang, Y. X. \& Rudnicki, M. A. Building muscle: molecular regulation of myogenesis. Cold Spring Harb. Perspect. Biol. 4, a008342 (2012).

3. Braun, T. \& Gautel, M. Transcriptional mechanisms regulating skeletal muscle differentiation, growth and homeostasis. Nat. Rev. Mol. Cell Biol. 12, 349-361 (2011).

4. Forcina, L., Miano, C., Pelosi, L. \& Musaro, A. An overview about the biology of skeletal muscle satellite cells. Curr. Genomics 20, 24-37 (2019).

5. Feige, P., Brun, C. E., Ritso, M. \& Rudnicki, M. A. Orienting muscle stem cells for regeneration in homeostasis, aging, and disease. Cell Stem Cell 23, 653-664 (2018).

6. Urciuolo, A. et al. Collagen VI regulates satellite cell self-renewal and muscle regeneration. Nat. Commun. 4, 1964 (2013).

7. Rayagiri, S. S. et al. Basal lamina remodeling at the skeletal muscle stem cell niche mediates stem cell self-renewal. Nat. Commun. 9, 1075 (2018).

8. Baghdadi, M. B. et al. Notch-induced miR-708 antagonizes satellite cell migration and maintains quiescence. Cell Stem Cell 23, 859-868 (2018).

9. Troy, A. et al. Coordination of satellite cell activation and self-renewal by Parcomplex-dependent asymmetric activation of p38alpha/beta MAPK. Cell Stem Cell 11, 541-553 (2012).

10. Von Maltzahn, J., Bentzinger, C. F. \& Rudnicki, M. A. Wnt7a-Fzd7 signalling directly activates the Akt/mTOR anabolic growth pathway in skeletal muscle. Nat. Cell Biol. 14, 186-191 (2011).

11. Yue, F. et al. Pten is necessary for the quiescence and maintenance of adult muscle stem cells. Nat. Commun. 8, 14328 (2017).

12. Kitajima, Y. et al. The ubiquitin-proteasome system is indispensable for the maintenance of muscle stem cells. Stem Cell Rep. 11, 1523-1538 (2018).

13. Peters, J. M. The anaphase promoting complex/cyclosome: a machine designed to destroy. Nat. Rev. Mol. Cell Biol. 7, 644-656 (2006).

14. Pines, J. Cubism and the cell cycle: the many faces of the APC/C. Nat. Rev. Mol. Cell Biol. 12, 427-438 (2011).

15. Kim, S. \& Yu, H. Mutual regulation between the spindle checkpoint and APC/C. Semin. Cell Dev. Biol. 22, 551-558 (2011).

16. Park, H. J., Costa, R. H., Lau, L. F., Tyner, A. L. \& Raychaudhuri, P. Anaphasepromoting complex/cyclosome- $\mathrm{CDH} 1$-mediated proteolysis of the forkhead 
box $\mathrm{M} 1$ transcription factor is critical for regulated entry into $\mathrm{S}$ phase. Mol. Cell Biol. 28, 5162-5171 (2008).

17. Laoukili, J., Alvarez-Fernandez, M., Stahl, M. \& Medema, R. H. FoxM1 is degraded at mitotic exit in a Cdh1-dependent manner. Cell Cycle 7, 2720-2726 (2008).

18. Bella, L., Zona, S., Nestal, D. M. G. \& Lam, E. W. FOXM1: A key oncofoetal transcription factor in health and disease. Semin. Cancer Biol. 29, 32-39 (2014).

19. Kwok, C. T. et al. The Forkhead box transcription factor FOXM1 is required for the maintenance of cell proliferation and protection against oxidative stress in human embryonic stem cells. Stem Cell Res. 16, 651-661 (2016).

20. Hou, Y. et al. The transcription factor Foxm1 is essential for the quiescence and maintenance of hematopoietic stem cells. Nat. Immunol. 16, 810-818 (2015).

21. Chen, Z. et al. Forkhead Box M1 transcriptionally regulates the expression of long noncoding RNAs Snhg8 and Gm26917 to promote proliferation and survival of muscle satellite cells. Stem Cells 36, 1097-1108 (2018).

22. Pandit, B. \& Gartel, A. L. Mutual regulation of FOXM1, NPM and ARF proteins. J. Cancer 6, 538-541 (2015).

23. Kongsema, M. et al. RNF168 cooperates with RNF8 to mediate FOXM1 ubiquitination and degradation in breast cancer epirubicin treatment. Oncogenesis 5, e252 (2016)

24. Kim, S. H. et al. EZH2 protects glioma stem cells from radiation-induced cell death in a MELK/FOXM1-dependent manner. Stem Cell Rep. 4, 226-238 (2015).

25. Chen, Y. et al. Wnt-induced deubiquitination FoxM1 ensures nucleus betacatenin transactivation. EMBO J. 35, 668-684 (2016).

26. Zhang, N. et al. FoxM1 promotes beta-catenin nuclear localization and controls Wnt target-gene expression and glioma tumorigenesis. Cancer Cell $\mathbf{2 0}$ 427-442 (2011).

27. Nusse, R. \& Clevers, H. Wnt/beta-catenin signaling, disease, and emerging therapeutic modalities. Cell 169, 985-999 (2017).

28. Rudolf, A. et al. beta-Catenin activation in muscle progenitor cells regulates tissue repair. Cell Rep. 15, 1277-1290 (2016).

29. Wang, X., Kiyokawa, H., Dennewitz, M. B. \& Costa, R. H. The Forkhead Box m1b transcription factor is essential for hepatocyte DNA replication and mitosis during mouse liver regeneration. Proc. Natl Acad. Sci. USA 99, 16881-16886 (2002).

30. Hayashi, S., Manabe, I., Suzuki, Y., Relaix, F. \& Oishi, Y. KIf5 regulates muscle differentiation by directly targeting muscle-specific genes in cooperation with MyoD in mice. Elife 5, e17462 (2016).

31. Zhu, P. et al. The transcription factor Slug represses p16(Ink4a) and regulates murine muscle stem cell aging. Nat. Commun. 10, 2568 (2019).

32. Von Maltzahn, J., Chang, N. C., Bentzinger, C. F. \& Rudnicki, M. A. Wnt signaling in myogenesis. Trends Cell Biol. 22, 602-609 (2012).

33. Parisi, A. et al. APC is required for muscle stem cell proliferation and skeletal muscle tissue repair. J. Cell Biol. 210, 717-726 (2015)

34. Yousif, N. G., Hadi, N. R. \& Hassan, A. M. Indocyanine Green-001 (ICG-001) attenuates Wnt/beta-catenin-induces myocardial injury following sepsis. J. Pharmacol. Pharmacother. 8, 14-20 (2017).
35. Nigg, E. A. Mitotic kinases as regulators of cell division and its checkpoints. Nat Rev. Mol. Cell Biol. 2, 21-32 (2001).

36. Nurse, P. Universal control mechanism regulating onset of $M$-phase. Nature 344, 503-508 (1990).

37. Matthess, Y., Raab, M., Sanhaji, M., Lavrik, I. N. \& Strebhardt, K. Cdk1/cyclin B1 controls Fas-mediated apoptosis by regulating caspase-8 activity. Mol. Cell. Biol. 30, 5726-5740 (2010).

38. Kimata, Y. APC/C ubiquitin ligase: coupling cellular differentiation to G1/G0 phase in multicellular systems. Trends Cell Biol. 29, 591-603 (2019).

39. Kim, W. et al. Hippo signaling is intrinsically regulated during cell cycle progression by APC/C(Cdh1). Proc. Natl Acad. Sci. USA 116, 9423-9432 (2019).

40. Cheng, Y. Y. et al. Reprogramming-derived gene cocktail increases cardiomyocyte proliferation for heart regeneration. EMBO Mol. Med. 9, 251-264 (2017).

41. Tschuor, C. et al. Constitutive androstane receptor (Car)-driven regeneration protects liver from failure following tissue loss. J. Hepatol. 65, 66-74 (2016).

42. Chen, X. et al. The forkhead transcription factor FOXM1 controls cell cycledependent gene expression through an atypical chromatin binding mechanism. Mol. Cell. Biol. 33, 227-236 (2013).

43. Blanco-Bose, W. E. et al. C-Myc and its target FoxM1 are critical downstream effectors of constitutive androstane receptor (CAR) mediated direct liver hyperplasia. Hepatology 48, 1302-1311 (2008).

44. Ngan, A. et al. Novel nuclear partnering role of EPS8 with FOXM1 in regulating cell proliferation. Front. Oncol. 9, 154 (2019).

45. Chai, N. et al. FOXM1 promotes proliferation in human hepatocellular carcinoma cells by transcriptional activation of CCNB1. Biochem. Biophys. Res. Commun. 500, 924-929 (2018).

46. Saldivar, J. C. et al. An intrinsic S/G2 checkpoint enforced by ATR. Science 361, 806-810 (2018).

47. Jang, S. H., Kim, A. R., Park, N. H., Park, J. W. \& Han, I. S. DRG2 regulates G2/M progression via the Cyclin B1-Cdk1 complex. Mol. Cells 39, 699-704 (2016).

48. Chen, Y. J. et al. A conserved phosphorylation site within the forkhead domain of FoxM1B is required for its activation by cyclin-CDK1. J. Biol. Chem. 284, 30695-30707 (2009).

49. Park, H. J., Costa, R. H., Lau, L. F., Tyner, A. L. \& Raychaudhuri, P. Anaphasepromoting complex/cyclosome-CDH1-mediated proteolysis of the forkhead box $\mathrm{M} 1$ transcription factor is critical for regulated entry into S phase. Mol. Cell. Biol. 28, 5162-5171 (2008)

50. Myatt, S. S. et al. SUMOylation inhibits FOXM1 activity and delays mitotic transition. Oncogene 33, 4316-4329 (2014).

51. Li, L. Q., Pan, D., Chen, H., Zhang, L. \& Xie, W. J. F-box protein FBXL2 inhibits gastric cancer proliferation by ubiquitin-mediated degradation of forkhead box M1. FEBS Lett. 590, 445-452 (2016)

52. Wang, $X$. et al. VprBP/DCAF1 regulates the degradation and nonproteolytic activation of the cell cycle transcription factor FoxM1. Mol. Cell. Biol. 37, 16 (2017). 